\title{
An organic electronic biomimetic neuron enables auto-regulated neuromodulation
}

\author{
Daniel Simon, Karin C. Larsson, David Nilsson, Gustav Burstrom, Dagmar Galter, Magnus \\ Berggren and Agneta Richter-Dahlfors
}

\section{Linköping University Post Print}

\section{Tweet}

N.B.: When citing this work, cite the original article.

Original Publication:

Daniel Simon, Karin C. Larsson, David Nilsson, Gustav Burstrom, Dagmar Galter, Magnus Berggren and Agneta Richter-Dahlfors, An organic electronic biomimetic neuron enables autoregulated neuromodulation, 2015, Biosensors \& bioelectronics, (71), 359-364.

http://dx.doi.org/10.1016/j.bios.2015.04.058

Copyright: Elsevier http://www.elsevier.com/

Postprint available at: Linköping University Electronic Press http://urn.kb.se/resolve?urn=urn:nbn:se:liu:diva-120203 


\section{An organic electronic biomimetic neuron enables auto- regulated neuromodulation}

Daniel T. Simon, ${ }^{\text {abc }}$ Karin C. Larsson, ${ }^{\text {ab }}$ David Nilsson, ${ }^{\text {d }}$ Gustav Burström, ${ }^{\text {ab }}$ Dagmar Galter, ${ }^{\text {b }}$ Magnus Berggren, ${ }^{\mathrm{c}}$ and Agneta Richter-Dahlfors* ${ }^{\mathrm{ab}}$

${ }^{a}$ Swedish Medical Nanoscience Center, Karolinska Institutet, S-171 77 Stockholm, Sweden.

${ }^{\mathrm{b}}$ Department of Neuroscience, Karolinska Institutet, S-171 77 Stockholm, Sweden.

${ }^{c}$ Laboratory of Organic Electronics, Department of Science and Technology, Linköping University, S-601 74 Norrköping, Sweden.

d Acreo Swedish ICT AB, S-602 21 Norrköping, Sweden.

* Corresponding authors:

Daniel T. Simon

Laboratory of Organic Electronics, ITN, Linköping University, S-601 74 Norrköping, Sweden +46(0) 11363476 daniel.simon@liu.se fax: +46(0) 11363270

Agneta Richter-Dahlfors

Swedish Medical Nanoscience Center, Karolinska Institutet, S-171 77 Stockholm, Sweden +46(0) 852487425 agneta.richter.dahlfors@ki.se fax: +46(0) 8311101 


\section{Abstract}

Current therapies for neurological disorders are based on traditional medication and electric stimulation. Here, we present an organic electronic biomimetic neuron, with the capacity to precisely intervene with the underlying malfunctioning signalling pathway using endogenous substances. The fundamental function of neurons, defined as chemical-to-electrical-to-chemical signal transduction, is achieved by connecting enzyme-based amperometric biosensors and organic electronic ion pumps. Selective biosensors transduce chemical signals into an electric current, which regulates electrophoretic delivery of chemical substances without necessitating liquid flow. Biosensors detected neurotransmitters in physiologically relevant ranges of 5-80 $\mu \mathrm{M}$, showing linear response above $20 \mu \mathrm{m}$ with approx. $0.1 \mathrm{nA} / \mu \mathrm{M}$ slope. When exceeding defined threshold concentrations, biosensor output signals, connected via custom hardware/software, activated local or distant neurotransmitter delivery from the organic electronic ion pump. Changes of $20 \mu \mathrm{M}$ glutamate or acetylcholine triggered diffusive delivery of acetylcholine, which activated cells via receptor-mediated signalling. This was observed in real-time by single-cell ratiometric $\mathrm{Ca}^{2+}$ imaging. The results demonstrate the potential of the organic electronic biomimetic neuron in therapies involving long-range neuronal signalling by mimicking the function of projection neurons. Alternatively, conversion of glutamate-induced descending neuromuscular signals into acetylcholine-mediated muscular activation signals may be obtained, applicable for bridging injured sites and active prosthetics.

\section{Keywords}

Neuromodulation, Organic electronic material, controlled drug release, neural prosthesis 


\section{Introduction}

Disorders of neural function often involve abnormal electrical and neurochemical signalling in the central nervous system. To improve quality of life, patients undergo a variety of neuromodulation therapies. Deep brain stimulation is a commonly applied electrical-technique with proven beneficial effects, despite some lack of understanding of its molecular mechanism of action (Kringelbach et al., 2007; Leeman and Cole, 2008; Olanow et al., 2004; Singh et al., 2007). Localized drug delivery can also be used, by means of fluidic systems (Rossi et al., 2013; Whitesides, 2006). However, these are often encumbered by accessory equipment (i.e., tubes, pumps, valves) and have difficulty in determining precise dosage.

Organic electronic electrophoretic transport devices - so called, iontronics - represent a new class of technologies that can provide spatiotemporal resolution and biochemical specificity. Based on polyelectrolytes and $\pi$-conjugated semiconducting polymers, iontronics exhibit a unique combination of ionic and electronic properties, enabling transduction between electronic impulses and biochemical signals (Larsson et al., 2013). This was first demonstrated in the organic electronic ion pump (OEIP), an electrophoretic delivery system which transports ions independently of liquid flow (Isaksson et al., 2007). The technology is based on thin films of the electrically conducting polymer poly(3,4-ethylenedioxythiophene) (PEDOT) doped with the polyelectrolyte poly(styrenesulfonate) (PSS) (Heywang and Jonas, 1992) to provide enhanced electronic conductivity as well as cation-selective ionic conductivity. An OEIP consists of two PEDOT:PSS electrode strips on a plastic substrate, each passing through an electrolyte-filled reservoir (Fig. S1a). One end of each strip is connected to a power supply, while immersion of the other ends in the target system forms a salt-bridge. When voltage is applied, positively charged substances in the source (anodic) reservoir are electrophoretically "pumped" into the target solution, without liquid flow. Simultaneously, cations are transported into the cathodic electrode/reservoir system, completing the electrochemical circuit. The precise electrochemistry of PEDOT:PSS enables delivery of one positively charged molecule for each electron involved in PEDOT:PSS redox, leading to extremely high dosage precision (Isaksson et al., 2007; Simon et al., 2009).

A neuron can be considered a chemical-to-electrical-to-chemical signal transduction unit (Fig. 1) able to convey information over long distances. Neurotransmitter binding incrementally depolarizes the cell membrane, generating an action potential of ionic currents at the axon hillock when threshold depolarization is reached. The action potential propagates along the cell membrane, leading to release of neurotransmitters at the axon terminal. Diffusion across the synaptic cleft enables neurotransmitter binding to receptors on the postsynaptic neuron. In this study, we developed an autoregulated electrochemical system, the organic electronic 
biomimetic neuron (OEBN), mimicking these fundamental aspects of neural function.

Chemical-to-electrical signal transduction is achieved by selective amperometric biosensors, which in turn regulate electrical-to-chemical delivery of neurotransmitters. This is the first demonstration of a regulated biosensor-OEIP system with the ability to precisely modulate delivery of neurotransmitters based on sensing of endogenous biochemical signals, thus mimicking neural function. The unique electronic communication interface allows the sensing component to be located at an arbitrary distance from the point of delivery, thereby expanding future applications to a variety of medical areas.

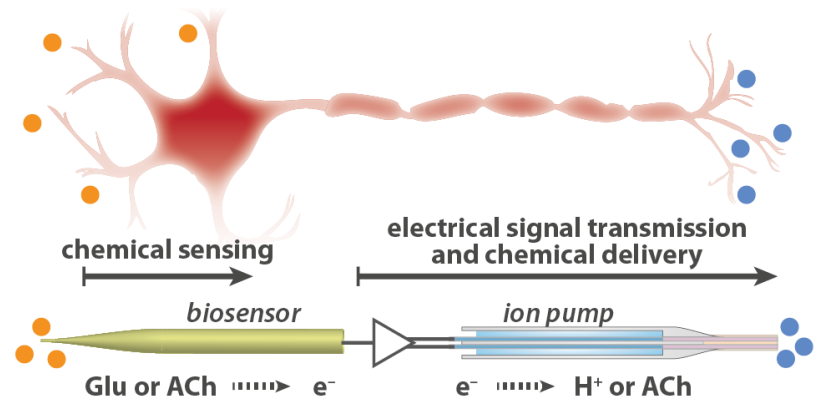

Figure 1. Chemical-to-electrical-to-chemical signal transmission of a neuron. A neuron (upper panel) integrates chemical signals (left), triggers an electrical pulse of membrane depolarization (action potential) along the axon, causing chemical release at the axon terminals (right). This functional signal transduction can be mimicked by a biosensor connected to an organic electronic ion pump, thereby forming an organic electronic biomimetic neuron (lower panel). Both systems comprise a chemical sensing component detecting a neuroactive species (orange circles), an electrical signal transmission region, and a chemical output component delivering another species (blue circles).

\section{Materials and methods}

\subsection{Manufacturing and operation of OEIP}

OEIP devices (Fig. S1) were manufactured by screen-printing PEDOT:PSS (Clevios SV3, H.C. Starck) onto poly-ethylene terephthalate sheets pre-coated with PEDOT:PSS (Orgacon EL-350, Agfa-Gaevert). Double-electrode strips were prepared, and mounted in two-lumen polypropylene tubes, serving as anodic and cathodic chambers. The length of the delivery tip in final devices was $\sim 7 \mathrm{~mm}$. Upon electronic addressing of PEDOT:PSS, the anode is oxidized (1), and positively charged species $\left(\mathrm{M}^{+}\right)$are liberated into the anode-side electrolyte. The cathode is reduced (2) and any cationic species $\left(\mathrm{X}^{+}\right)$compensate the $\mathrm{PSS}^{-}$in the target system.

$$
\begin{aligned}
& \text { PEDOT }^{0}+\mathrm{M}^{+}: \text {PSS }^{-} \longrightarrow \text { PEDOT }^{+}: \text {PSS }^{-}+\mathrm{M}^{+}+\mathrm{e}^{-} \\
& \text {PEDOT }^{+}: \text {PSS }^{-}+\mathrm{X}^{+}+\mathrm{e}^{-} \longrightarrow \text { PEDOT }^{0}+\mathrm{X}^{+}: \text {PSS }^{-}
\end{aligned}
$$


The electrochemical potential established between the electrodes electrophoretically "pumps" the liberated $\mathrm{M}^{+}$into the target system, and $\mathrm{X}^{+}$is transported in toward the cathode. Devices were operated as described in Supplementary Materials and Methods.

\subsection{Real-time $\mathrm{pH}$ recording}

An OEIP filled with $100 \mathrm{mM} \mathrm{HCl(aq)} \mathrm{was} \mathrm{immersed} \mathrm{into} \mathrm{a} \mathrm{dish} \mathrm{containing} 100 \mathrm{mM} \mathrm{NaCl}(\mathrm{aq})$ and universal $\mathrm{pH}$ indicator (Fluka 36828) mounted on the stage of a stereo-microscope (Nikon SMZ1500). Color change at the submerged delivery tip was recorded by time-lapse video. $\mathrm{pH}$ was approximated by comparing the blue channel intensity of the video over time with the calibration colors of the indicator (Isaksson et al., 2008).

\subsection{Chemical sensing by amperometric biosensors}

Commercial amperometric enzymatic biosensors utilize enzymatic reactions that oxidize or reduce the chemical component to be detected. This generates electrons available for electrical recording. The highly selective Glu sensor (7001, Pinnacle Technology) yields exactly two electrons for each Glu $(3,4)$, and provides a sensor current directly proportional to [Glu].

$$
\begin{aligned}
& \text { Glu }+\mathrm{O}_{2}+\mathrm{H}_{2} \mathrm{O} \stackrel{\text { GluOx }}{\longrightarrow} \text { 2-oxoglutarate }+\mathrm{NH}_{3}+\mathrm{H}_{2} \mathrm{O}_{2} \\
& \mathrm{H}_{2} \mathrm{O}_{2} \stackrel{500 \mathrm{mV}}{\longrightarrow} 2 \mathrm{H}^{+}+\mathrm{O}_{2}+2 \mathrm{e}^{-}
\end{aligned}
$$

The ACh sensor (Sarissaprobe ACh, Sarissa Biomedical) yield four electrons from each ACh (5-7), and determines changes in [ACh] in environments lacking choline or with static [choline].

$$
\begin{aligned}
& \text { ACh }+\mathrm{H}_{2} \mathrm{O} \stackrel{\text { AChE }}{\longrightarrow} \text { acetate }+ \text { choline } \\
& \text { choline }+\mathrm{O}_{2} \stackrel{\text { ChOx }}{\longrightarrow} \text { betaine aldehyde }+2 \mathrm{H}_{2} \mathrm{O}_{2} \\
& 2 \mathrm{H}_{2} \mathrm{O}_{2} \stackrel{500 \mathrm{mV}}{\longrightarrow} 4 \mathrm{H}^{+}+2 \mathrm{O}_{2}+4 \mathrm{e}^{-}
\end{aligned}
$$

The Glu sensor was operated at $600 \mathrm{mV}$ versus the built-in $\mathrm{Ag} / \mathrm{AgCl}$ wire reference, and the $\mathrm{ACh}$ sensor at $500 \mathrm{mV}$ versus an external $\mathrm{Ag} / \mathrm{AgCl}$ reference electrode (Bioanalytical Systems). Voltage was applied and current recorded using a Keithley 2602 SourceMeter and custom LabVIEW software. 


\subsection{Cell cultivation and intracellular $\mathrm{Ca}^{2+}$ recordings}

Human neuroblastoma SH-SY5Y cells (ATCC no. CRL-2266) were handled according to supplier's instruction, and ratiometric $\mathrm{Ca}^{2+}$ imaging was performed as previously described (Tybrandt et al., 2009) and detailed in the Supplementary Materials and Methods.

\section{Results}

\subsection{Amperometric biosensors to determine activation thresholds}

Neurons sense small concentration changes in the neurochemical environment and if threshold concentrations are reached, initiate action potentials. To mimic such sensing, real-time monitoring of [Glu] or [ACh] was demonstrated using commercially available, highly selective enzyme-based amperometric biosensors. To ascertain their ability to operate in complex cell medium, a Glu biosensor was first mounted in a dish containing Glu-free cell medium and the baseline sensor current was allowed to stabilize. [Glu] was then increased stepwise from 5-80 $\mu \mathrm{M}$, matching the concentration range reported for the synaptic cleft $(2 \mu \mathrm{M}-1 \mathrm{mM})$, brain extracellular fluid, and blood plasma (Meldrum, 2000). With each increase, the biosensor current rose sharply within the first few seconds, before equilibrating at a level corresponding to the concentration step (Fig. 2a). These peaks never exceeded the equivalent of an approximately $20 \mu \mathrm{M}$ overestimation of [Glu], permitting threshold concentrations to be set with $20 \mu \mathrm{M}$ resolution. After equilibration at $20 \mu \mathrm{M}$ Glu, the change in sensor current was linear and approximately $1 \mathrm{nA}$ for each $10 \mu \mathrm{M}$ rise in [Glu].

ACh sensing was achieved by an ACh-specific biosensor. Stepwise [ACh] increase from $0-80$ $\mu \mathrm{M}$ raised the biosensor current accordingly (Fig. 2b). The maximum transient overestimation (on the step to $80 \mu \mathrm{M} \mathrm{ACh}$ ) did not exceed $\sim 5 \mu \mathrm{M}$ and equilibrium was generally reached faster as compared to the Glu sensor. Each $10 \mu \mathrm{M}$ rise in [ACh] corresponded to an approximately $700 \mathrm{pA}$ rise in the current. Together, these experiments demonstrate that the biosensors were suitable for sensing concentration changes in complex cell media and translating them into electric currents, regardless of potentially electrochemically-active interferents in the cell media.
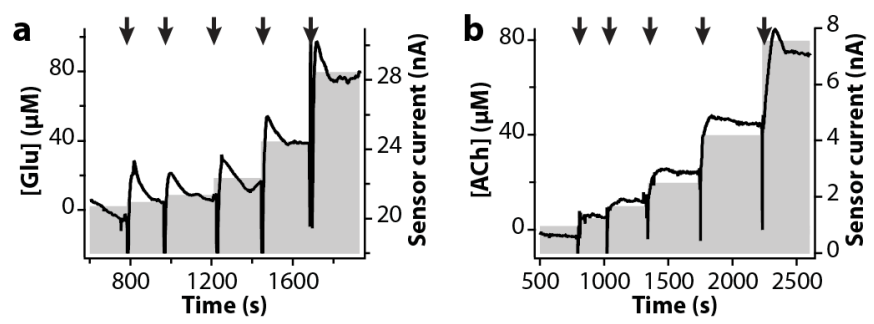

Figure 2. Amperometric biosensing of Glu and ACh. Chemical signals, represented by (a) Glu and (b) ACh, are sensed and transduced into electrical signals (current, right axis). Grey bars indicate the manually adjusted 
concentration (left axis), arrows indicate the time points of these adjustments. Each experiment was conducted a minimum of three times.

\subsection{Threshold-induced activation of chemical delivery}

Having established detection and translation of chemical signals into electrical currents, we coupled the biosensor to the OEIP to achieve a system for auto-regulated chemical delivery. Electronically controlled transport and delivery through the tip of the OEIP is accomplished without any liquid flow, thereby mimicking the neuron's diffusive release of neurotransmitters. To visualize this local release, we loaded an OEIP with $100 \mathrm{mM} \mathrm{HCl(aq)}$ and recorded the spatially resolved delivery of $\mathrm{H}^{+}$(Isaksson et al., 2008) in a dish containing universal $\mathrm{pH}$ indicator in $10 \mathrm{ml} 100 \mu \mathrm{M} \mathrm{NaCl}(\mathrm{aq})$ (Fig. 3a). A Glu biosensor, submerged in a separate sensing dish containing $10 \mathrm{ml}$ PBS, was connected to the OEIP via control hardware and software. [Glu] was increased stepwise in the sensing dish and the biosensor current was recorded (Fig. 3b). Increasing [Glu] to $10 \mu \mathrm{M}$ gave a sensor current of $6.3 \mathrm{nA}$, and further increase to $20 \mu \mathrm{M}$ gave $9.2 \mathrm{nA}$. Both current levels were well below the arbitrarily defined threshold for OEIP activation set at $20 \mathrm{nA}$ (corresponding to [Glu] $>40 \mu \mathrm{M}$ ). After exceeding the threshold current by increasing [Glu] to $80 \mu \mathrm{M}$, we next diluted [Glu] to $40 \mu \mathrm{M}$ and observed that the sensor current dropped accordingly.
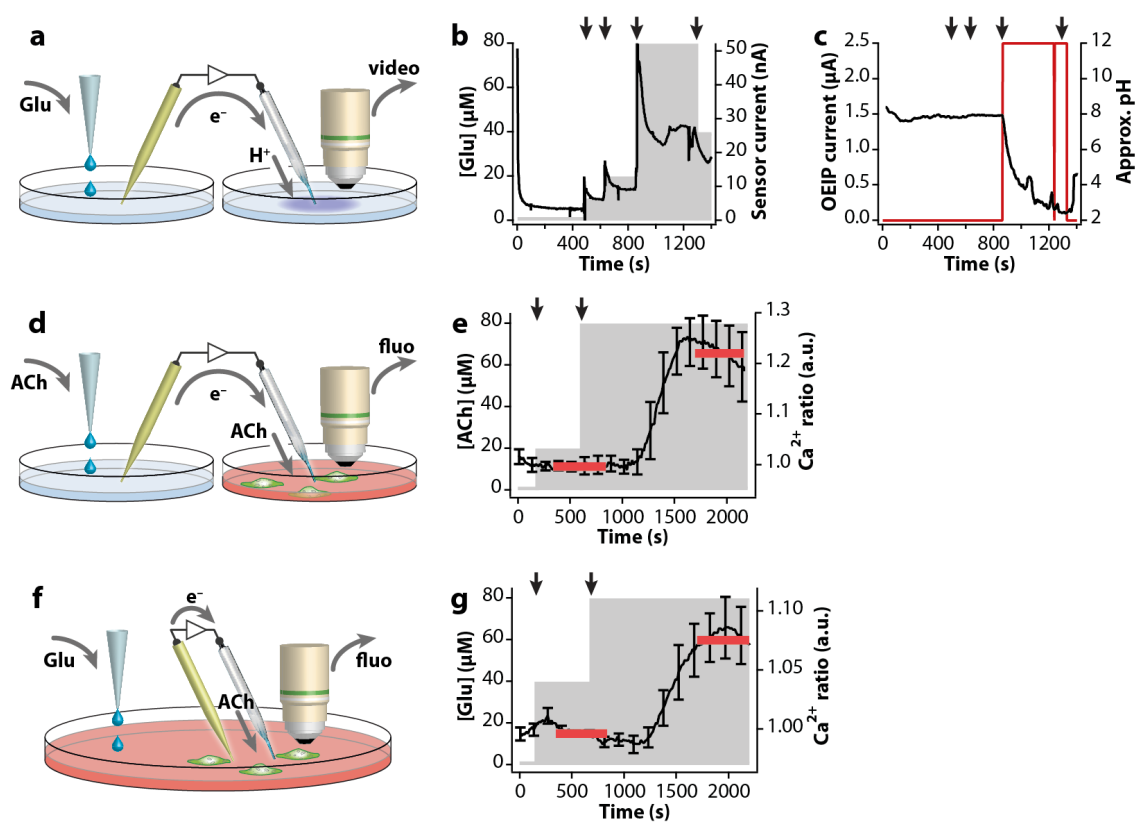

Figure 3. OEBN enables chemical-to-electrical-to-chemical signal transduction. (a) Set-up showing manual Glu addition (drops) to a dish with a Glu biosensor (green). Glu sensing generates electronic signals ( $\left.\mathrm{e}^{-}\right)$, which via hard/software regulates $\mathrm{H}^{+}$delivery from the OEIP (white tube) in a target dish. $\mathrm{pH}$ is monitored microscopically (video). (b) Sensor current as a function of increasing [Glu] (grey bars), arrows indicate time of adjustments in the sensor dish. (c) ON/OFF state of OEIP delivery current (red) regulates pH (black) near the delivery tip in the target dish. Arrows same as in (b). See also Supp. Movie 1 and Fig. S2. (d) Long-range ACh-to-ACh signalling achieved by transducing manually added ACh in left dish into automated ACh delivery to cells in right dish, observed by 
fluorescence-based $\mathrm{Ca}^{2+}$ recording (fluo). (e) $\mathrm{Ca}^{2+}$ signalling in cells (right dish) in response to altered [ACh] (grey bars) in left dish. Responses, including standard deviation, shown for an average of 15 cells/cluster. Red lines indicate average $\mathrm{Ca}^{2+}$ response over $500 \mathrm{~s}$ spans before and after ACh activation, aiding to visualise the effect of ACh delivery. Arrows indicate time for [ACh] adjustments in left dish. (f) Local Glu-to-ACh signalling in single dish. Manually added Glu is transduced into automated ACh delivery, while cellular $\mathrm{Ca}^{2+}$ response (fluo) is observed. (g) Intracellular $\mathrm{Ca}^{2+}$ signalling in response to altered [Glu] (grey bars). Responses, including standard deviation, shown for an average of 9 cells/clusters. Red lines and arrows as in (e). See also Supp. Movie 2.

The current recorded from the biosensor was used as input signal to the OEIP control software. While the sensor current remained below threshold, the OEIP remained OFF and $\mathrm{pH}$ in the target dish stayed constant at $\mathrm{pH} 8$ (Fig. 3c). Exceeding the threshold ( $80 \mu \mathrm{M}$ Glu at $860 \mathrm{~s})$ turned the OEIP current $\mathrm{ON}$, initiating $\mathrm{H}^{+}$delivery. This is observed as an immediate decrease of the $\mathrm{pH}$ in the target dish. The $\mathrm{pH}$ continued to decrease from 8 to $<3$ during OEIP activation at $2.5 \mu \mathrm{A}$ delivery current, which remained constant except for a momentary OFF switch due to agitation of the solution at the biosensor. Diluting Glu, resulting in a sensor current below threshold, caused the software to turn the OEIP OFF. This terminated $\mathrm{H}^{+}$delivery and the $\mathrm{pH}$ rose toward neutral due to the rapid diffusion of $\mathrm{H}^{+}$in the PBS. Supplementary Movie 1 shows a video recording of the OEIP tip during this experiment, and Fig. S2 contains several still images illustrating characteristic behaviour. By converting Glu fluxes into $\mathrm{H}^{+}$fluxes in this way, these data demonstrate a chemical-to-electrical-to-chemical signalling system.

\subsection{Mimicking neurons in vitro: distant delivery and cell activation}

In our development towards an auto-regulated neuromodulatory system, we next delivered the excitatory neurotransmitter $\mathrm{ACh}$, a small positively charged molecule with high transport efficiency in the OEIP (Tybrandt et al., 2009). As readout, adherent SH-SY5Y cells expressing ACh-receptors (AChRs) were used, since their binding of ACh can be detected by real-time $\mathrm{Ca}^{2+}$ imaging. An OEIP filled with ACh was mounted with its delivery tip adjacent to cells loaded with the $\mathrm{Ca}^{2+}$ dye Fura-2 AM (Fig. 3d). The OEIP was connected through the control hard/software to an ACh biosensor in a separate dish. The software was programmed to initiate ACh delivery to the cells when the biosensor detected $[\mathrm{ACh}] \geq 80 \mu \mathrm{M}$. After calibrating the $\mathrm{ACh}$ biosensor and allowing the current to stabilize, the baseline current was recorded in parallel with the basal intracellular $\mathrm{Ca}^{2+}$ fluorescence (Fig. 3e). An increase of [ACh] to $20 \mu \mathrm{M}$ was insufficient to trigger OEIP delivery since it was below the arbitrarily set threshold. Accordingly, no $\mathrm{Ca}^{2+}$ response was observed. When increasing [ACh] to $80 \mu \mathrm{M}$, the preprogrammed threshold was exceeded. Approximately $500 \mathrm{~s}$ later, an increase in intracellular $\left[\mathrm{Ca}^{2+}\right]$ was observed as a shift in the fluorescence ratio. This experiment demonstrated how ACh fluxes at one site can be converted into ACh fluxes at a remote point. 


\subsection{Mimicking neurons in vitro: local sensing and cell activation}

To demonstrate a more unified design of the auto-regulated system, the biosensor and OEIP were positioned in the same dish, adjacent to each other (Fig. 3f). To demonstrate sensing and recording in close proximity within the same dish, Glu was used as biosensor input since SHSY5Y cells are unresponsive due to incomplete expression of Glu receptors (Fig. S3 and (Kulikov et al., 2007)). ACh was used as the delivery substance for cell stimulation. A micromanipulator was used to position the Glu biosensor and the OEIP approximately $10 \mathrm{~mm}$ apart, leaving space for the microscope objective used to monitor the intracellular $\mathrm{Ca}^{2+}$ response. The control software was programmed to initiate OEIP delivery of ACh when [Glu] exceeded $80 \mu \mathrm{M}$. When concurrent recordings of biosensor current and basal intracellular $\mathrm{Ca}^{2+}$ fluorescence had stabilized (Fig. 3g), [Glu] was increased to $40 \mu \mathrm{M}$. Still not exceeding the [Glu] threshold, the OEIP remained in the OFF state. Next, an increase to $80 \mu \mathrm{M}$ exceeded the threshold, switching the OEIP ON. The resulting ACh delivery activated AChRs, leading to an increased intracellular $\left[\mathrm{Ca}^{2+}\right]$ after approx. 500 s (Fig. $3 \mathrm{~g}$ and Supplementary Movie 2).

Based on modelling of ACh delivery (Fig. S4), this approx. $500 \mathrm{~s}$ delay seen in Fig. 3e and $3 \mathrm{~g}$ can be attributed to the time required for electromigration of ACh through the OEIP channel, and to reach the threshold [ACh] in the target electrolyte. While the observed $\mathrm{Ca}^{2+}$ responses are slow compared to neuronal activity, recent developments with planar OEIPs (Tybrandt et al., 2009) into future OEBNs will significantly enhance the temporal dynamics, approaching the time scale of neurons. Another current limitation is the slight variation in the three-dimensional positioning of the delivery tip. While we strove to position the tip as close to the cells as possible, the lateral and vertical distance may have varied between experiments.

\section{Discussion}

By combining a chemical sensing component with an OEIP delivery device, we have demonstrated the concept of an organic electronic biomimetic neuron (OEBN). The OEBN demonstrates the threshold-response of action potentials and chemical-to-electrical-to-chemical signalling, both hallmarks of neurons. The chemical sensing component, comprising off-theshelf biosensors, can be exchanged for any biosensor system capable of outputting an electrical signal: other commercially available sensors or custom made higher-performance technologies (Kergoat et al., 2012). An increasing availability of miniaturized, selective and sensitive electrochemical biosensors for numerous chemical compounds (Marinesco and Dale, 2013; Turner, 2013) further expands future medical applications. 
The chemical delivery component, based on organic polyelectrolytes and $\pi$-conjugated conducting polymers, exhibits advantages such as flexibility, rheological compatibility with tissue, and ease of fabrication (Martin et al., 2010). The electrochemical nature of delivery enables fine-tuned temporal control by prompt ON/OFF switching - a unique feature amongst delivery devices. The repertoire of deliverable substances, currently including mono- and divalent cations (Isaksson et al., 2007), negatively charged species (Tybrandt et al., 2010), aspartate, Glu, GABA (Simon et al., 2009) and ACh (Tybrandt et al, 2009), is likely to expand as material properties are being further developed.

Although current limitations, such as response time and size, prohibit true synaptic integration in its current format, OEBN technology can be envisaged in future therapeutic applications. This includes long-distance communication, similar to projection neurons with very long axons, communicating with neurons in distant areas of the nervous system. This prefigures addition of $\mathrm{ACh}$ when endogenous [ACh] at distant sites drop too low. Abnormalities of [ACh] in the forebrain are strongly implicated in Alzheimer's disease, and therapies based on enhanced [ACh] are being explored (Mayeux and Sano, 1999). Other applications involve therapies requiring signal translation, i.e., converting Glu-induced descending neuromuscular signals into ACh-mediated muscular activation signals at neuromuscular junctions. The custom-designed length of the electrical communication component, potentially replaced by wireless communication, will further expand the use of OEBN in bridging injured sites and in active prosthetics.

\section{Conclusions}

We presented a biomimetic device able to reproduce the chemical-to-electrical-to-chemical signal transduction function of neurons. The organic electronic biomimetic neuron enabled neurotransmitter-regulated signaling over short and long distances, and effectively translated the sensing of one neurotransmitter into the release of another. This versatility offers a novel means for auto-regulated neuromodulation based on endogenous substances, enabling malfunctioning neuronal signalling pathways to be restored or augmented by the same chemical queues as present in the healthy state.

\section{Acknowledgements}

We thank A. Eveborn and A. Malmström for help in fabricating OEIPs, Dr. S. Löffler for experimental help. Strategic Research Center for Organic Bioelectronics (www.oboecenter.se, funded by the Swedish Foundation for Strategic Research and VINNOVA), and the Swedish Medical Nanoscience Center (www.medicalnanoscience.se) funded by Carl Bennet AB, 
VINNOVA, and Karolinska Institutet supported the project, as well as the Swedish Research

Council, Swedish Brain Power, KAW, Royal Swedish Academy of Sciences, and Önnesjö

Foundation.

\section{References}

Heywang, G., Jonas, F., 1992. Adv. Mater. 4, 116-118.

Isaksson, J., Kjäll, P., Nilsson, D., Robinson, N., Berggren, M., Richter-Dahlfors, A., 2007. Nat. Mater. 6, 673-679.

Isaksson, J., Nilsson, D., Kjäll, P., Robinson, N.D., Richter-Dahlfors, A., Berggren, M., 2008. Org. Electron. 9, 303-309.

Kergoat, L., Piro, B., Berggren, M., Horowitz, G., Pham, M.-C., 2012. Anal. Bioanal. Chem. 402, 1813-1826.

Kringelbach, M.L., Jenkinson, N., Owen, S.L.F., Aziz, T.Z., 2007. Nat. Rev. Neurosci. 8, 623635.

Kulikov, A.V., Rzhaninova, A.A., Goldshtein, D.V., Boldyrev, A.A., 2007. B. Exp. Biol. Med. $144,626-629$.

Larsson, K.C., Owens, R.M., Kjäll, P., Kjäll, P., Richter-Dahlfors, A., Cicoira, F., 2013. BBAGen. Subjects 1830, 4334-4344.

Leeman, B.A., Cole, A.J., 2008. Annu. Rev. Med. 59, 503-523.

Marinesco, S., Dale, N., 2013. Microelectrode Biosensors. Humana Press, Totowa, NJ.

Martin, D., Wu, J., Shaw, C., King, Z., Spanninga, S., Richardson-Burns, S., Hendricks, J., Yang, J., 2010. Polym. Rev. 50, 340-384.

Mayeux, R., Sano, M., 1999. New Engl. J. Med. 341, 1670-1679.

Meldrum, B., 2000. J. Nutr. 130, 1007S-1015S.

Olanow, C.W., Agid, Y., Mizuno, Y., Albanese, A., Bonucelli, U., Damier, P., De Yebenes, J., Gershanik, O., Guttman, M., Grandas, F., Hallett, M., Hornykiewicz, O., Jenner, P., Katzenschlager, R., Langston, W.J., LeWitt, P., Melamed, E., Mena, M.A., Michel, P.P., Mytilineou, C., Obeso, J.A., Poewe, W., Quinn, N., Raisman-Vozari, R., Rajput, A.H., Rascol, O., Sampaio, C., Stocchi, F., 2004. Movement Disord. 19, 997-1005.

Rossi, F., Perale, G., Papa, S., Forloni, G., Veglianese, P., 2013. Expert Opin. Drug Del. 10, 385-396.

Simon, D.T., Kurup, S., Larsson, K.C., Hori, R., Tybrandt, K., Goiny, M., Jager, E.W.H., Berggren, M., Canlon, B., Richter-Dahlfors, A., 2009. Nat. Mater. 8, 742-746.

Singh, N., Pillay, V., Choonara, Y.E., 2007. Prog. Neurobiol. 81, 29-44.

Turner, A.P.F., 2013. Chem. Soc. Rev. 42, 3184-3196.

Tybrandt, K., Larsson, K.C., Kurup, S., Simon, D.T., Kjäll, P., Isaksson, J., Sandberg, M., Jager, E.W.H., Richter-Dahlfors, A., Berggren, M., 2009. Adv. Mater. 21, 4442-4446.

Tybrandt, K., Larsson, K.C., Richter-Dahlfors, A., Berggren, M., 2010. P. Natl. Acad. Sci. 107, 9929-9932.

Whitesides, G.M., 2006. Nature 442, 368-373. 